REVUE DE L'INSTITUT

FRANÇAIS D'HISTOIRE

EN ALLEMAGNE

\section{Revue de l'IFHA}

Revue de l'Institut français d'histoire en Allemagne

$4 \mid 2012$

IFHA 4

\title{
Une histoire franco-allemande en onze volumes
}

\section{Pierre Monnet}

\section{OpenEdition}

\section{Journals}

Édition électronique

URL : http://journals.openedition.org/ifha/412

DOI : $10.4000 /$ ifha. 412

ISSN : 2198-8943

\section{Éditeur}

IFRA - Institut franco-allemand (sciences historiques et sociales)

\section{Édition imprimée}

Date de publication : 30 septembre 2012

Pagination : 85-90

ISSN : 2190-0078

\section{Référence électronique}

Pierre Monnet, « Une histoire franco-allemande en onze volumes », Revue de l'IFHA [En ligne], 4 | 2012,

mis en ligne le 14 février 2013, consulté le 03 mai 2019. URL : http://journals.openedition.org/ifha/412 ; DOI : 10.4000/ifha.412

Ce document a été généré automatiquement le 3 mai 2019.

(CIFHA 


\title{
Une histoire franco-allemande en onze volumes
}

\author{
Pierre Monnet
}

1 Depuis 2005, l'Institut historique allemand de Paris (IHAP) conduit, coordonne et finance l'édition d'une série d'ouvrages ambitieuse et unique en son genre : une histoire francoallemande en 11 volumes dirigée initialement par Werner Paravicini puis par son successeur à la tête de l'IHAP Gudrun Gersmann (IHAP Paris et université de Cologne) et par Michael Werner (EHESS/CIERA Paris). Si les premiers volumes sont parus en allemand, un accord passé avec les presses universitaires lilloises du Septentrion en 2010 prévoit la publication parallèle de chaque volume en français. Conformément à l'esprit de l'entreprise, le bilinguisme sera donc désormais assuré. Mais la double approche que commande l'idée même d'une histoire à cheval sur deux pays est également opérée à travers le choix des auteurs : 6 Allemands et 10 Français (certains volumes sont confiés à plusieurs spécialistes) ont été convoqués.

2 Ces deux dimensions du bilinguisme et de l'interculturalité constituent bien la garantie d'une histoire croisée des espaces français et allemand du haut Moyen Âge à nos jours, une histoire des connexions, des interférences, des circulations, sensible aux différences, aux ressemblances et aux interactions qui constituent donc autant de fenêtres ouvertes sur l'histoire de l'environnement européen et mondial dans lequel s'inscrivent naturellement les évolutions de chaque pays. Mais l'ambition de cette collection est également historiographique puisqu'elle entend dépasser le simple cadre chronologique et géopolitique pour s'intéresser aux échanges culturels au sens large ainsi qu'à la production et à la conservation des documents les plus adéquats pour parler des deux pays à la fois. À cet égard, le choix d'un seul fil directeur, fût-il spatial, politique ou linguistique, ne pouvait être fait pour rendre compte d'une histoire plurielle. Aussi, c'est dans la présentation même de la matière qu'une homogénéité a été retenue, imposant pour chaque volume un triptyque consistant à restituer dans un premier temps les grandes césures, les logiques territoriales, les équilibres et rapports de force, les échanges consentis ou forcés, avant de formuler dans un second temps quelques problématiques historiques actuelles dans la période couverte par chaque tome, pour fournir en troisième 
lieu une bibliographie synthétique, en français et en allemand, sans exclure la production en langue anglaise, russe, espagnole ou italienne selon les besoins. Un appareil cartographique, un index des noms de lieux et de personnes et un tableau chronologique viennent le plus souvent enrichir chaque volume.

Ce cadre de présentation commun n'empêche nullement chaque auteur de placer l'accent sur un point particulier et propre à la période, en fonction également des tendances les plus récentes de la recherche historique de chaque côté du Rhin. Le deuxième volume rédigé par Jean-Marie Moeglin fait suite au premier de la collection dans lequel Rolf Große analyse l'évolution du regnum français et de l'imperium allemand à partir d'un héritage franc commun ; J. M. M. part de la bataille de Bouvines en 1214 et insiste pour sa part sur l'importance des transferts culturels qui se sont produits entre les deux entités. Ces dernières commencent, sans doute par ce biais, à prendre conscience de leur identité, de leurs limites, de leurs frontières (à la fois politiques, territoriales et linguistiques), ce dernier terme formant un mot-clé de la réflexion. À l'autre bout de la chaîne, les deux derniers volumes de Corine Defrance et Ulrich Pfeil et de Hélène Miard-Delacroix se complètent en désacralisant tout d'abord la date mythique de 1963 (traité de l'Élysée), dont ils montrent bien l'esprit et les avancées, mais dont ils soulignent aussi les inachèvements et les lacunes. Ce faisant, ils redonnent droit aux évolutions sociales, culturelles et économiques plus profondes qui transforment radicalement les deux sociétés française et allemande de 1945 à nos jours (régimes et cultures politiques, pratique du parlementarisme, 1968 et sa place dans l'histoire, mutations de la société du travail, nationalité et citoyenneté) et replacent à raison la relation franco-allemande au cœur du processus de la construction européenne. L'un et l'autre volumes s'entendent finalement sur la thèse suivant laquelle, certes le moteur franco-allemand a été nécessaire à la construction européenne, mais qu'en retour ce couple n'aurait jamais pu fonctionner sans les convergences fondamentales et de haut niveau qui ont travaillé souterrainement les deux pays: la croissance puis la crise économique, les défis environnementaux et démographiques, la globalisation des échanges, l'explosion de la communication, la fin du bipolarisme mondial... C'est évidemment l'avantage, pour le lecteur, que de pouvoir lire d'affilée ces deux volumes pour en comprendre la continuité et la cohérence. C'est dans le même esprit que l'on peut tirer le meilleur parti de la succession des trois volumes couvrant la période moderne (1500-1648, 1648-1789 et 1789-1815) puisque l'on peut suivre d'un ouvrage à l'autre des thématiques communes telles que la dynamique des rapports entre monarchie nationale et Empire universalisant, l'interminable question des frontières, la force des représentations projetées par l'un sur le modèle ou l'anti-modèle de l'autre, l'importance croissante que tiennent la langue et l'histoire dans la définition de soi et du voisin. On regrettera seulement que, parmi ces thèmes de longue durée, la religion, les confessions, la piété, n'occupent pas la place que l'on aurait souhaité, tandis que si le volume central de la période moderne (1648-1789) ménage avec bonheur une part considérable aux échanges économiques, on ne retrouve pas avec autant d'importance le rôle de l'économie et du commerce (sans même parler de la monnaie) dans les deux volumes précédent et suivant. Un tel exercice de lecture continue n'est en revanche pas encore possible, en raison du calendrier de publication, pour le volume consacré à la période 1918-1933 (une chronologie allemande, mais comment pourrait-il en être autrement), dont on percevra seulement à plein les accents lorsque les volumes précédent (1971-1918) et suivant (1932-1945) seront disponibles. C'est en effet alors seulement que l'on pourra s'interroger avec raison sur la légitimité d'un partage de la période 1914-1945 en deux volumes, ou bien sur les raisons qui peuvent 
militer ou non en faveur du rattachement de la Première Guerre mondiale au volume s'ouvrant par 1871 plutôt qu'à celui conduisant au nazisme. En effet, si Nicolas Beaupré dans le volume 1918-1933 insiste à juste titre sur l'impossible paix, ou tout du moins sur les illusions de la paix, et consacre ensuite un chapitre à la «brutalisation » de la société d'après-guerre, il est difficile de ne pas voir que l'une et l'autre, la paix difficile d'une part, la violence de l'autre, qui dominent les années 1920-1930, sont déjà contenues dans l'épisode 1914-1918 et largement partagées par la France et par l'Allemagne avant comme après la guerre.

4 Les lecteurs allemands disposent à ce jour de 8 volumes parus : le premier (Rolf Große, Vom Frankenreich zu den Ursprüngen der Nationalstaaten : 800-1214, 2005), le deuxième (JeanMarie Moeglin, Kaisertum und allerchristlichster König: 1214-1500, 2010), le troisième (Rainer Babel, Deutschland und Frankreich im Zeichen der habsburgischen Universalmonarchie: 1500-1650, 2005), le quatrième (Guido Braun, Von der politischen zur kulturellen Hegemonie Frankreichs: 1648-1789, 2008), le cinquième (Bernhard Struck, Claire Gantet, Revolution, Krieg und Verflechtung: 1789-1815, 2008), le huitième (Nicolas Beaupré, Das Trauma des Großen Krieges 1918 bis 1932/33, 2009), le dixième (Corine Defrance, Ulrich Pfeil, Eine Nachkriegsgeschichte in Europa 1945 bis 1963, 2011) et le onzième (Hélène Miard-Delacroix, Im Zeichen der europäischen Einigung 1963 bis in die Gegenwart, 2011). Jusqu'en 2014 paraittront en allemand les trois derniers volumes : le sixième (Michael Werner, Nationen im Spiegelbild: 1815-1870), le septième (Mareike König, Elise Julien, Rivalités et interdépendances. France-Allemagne 1871-1918) et le neuvième (Johann Chapoutot, Alya Aglan, Jean-Michel Guien, Von der Krise in die Katastrophe : 1932-1945).

En version française sont jusqu'à présent parus trois volumes : le deuxième (Jean-Marie Moeglin, L'Empire et le royaume. Entre indifférence et fascination - 1214-1500, 2011), le dixième (Corine Defrance, Ulrich Pfeil, Entre guerre froide et intégration européenne. Reconstruction et rapprochement - 1945-1963, 2011) et le onzième (Hélène Miard-Delacroix, Le défi européen 1963 à nos jours, 2011). Dès l'automne 2012, les volumes quatre (Guido Braun, Du Roi-Soleil aux Lumières. L'Allemagne face à l'«Europe française » 1648 à 1789), cinq (Claire Gantet, Bernhard Struck, Révolution, guerre, interférences - 1789-1815) et huit (Nicolas Beaupré, Le traumatisme de la Grande Guerre - 1918-1932/33) viendront enrichir la série, rejoints en 2013 par les volumes un (Rolf Große, Du royaume franc aux origines de la France et de l'Allemagne 800-1214), trois (Rainer Babel, La France et l'Allemagne à l'époque de la monarchie universelle des Habsbourg - 1500-1648) et six (Michael Werner, Nations en miroirs: France et Allemagne 1815-1870), puis en 2014 par les volumes sept (Elise Julien, Mareike König, Rivalités et interdépendances - 1870-1918) et neuf (Johan Chapoutot, Alya Aglan, Jean-Michel Guieu, La paix impossible? De la crise à la catastrophe -1932-1945).

Quatre souhaits pourront au final être formulés. Le premier tient à l'absence d'une iconographie franco-allemande de l'histoire ainsi racontée. Mais on suppose que des contraintes éditoriales et financières ont dû peser pour limiter le recours à l'image. Le second tient à la fonction heuristique que pourrait remplir un lexique franco-allemand des termes historiques (notions, institutions...), que la parution parallèle dans les deux langues rend désormais possible et souhaitable. Le troisième tient à l'utilité que pourrait représenter une sorte de recueil des grands textes français et allemands auxquels se réfèrent constamment les grands récits délivrés au sein de chaque volume (traités, constitutions, portraits, descriptions de pays, romans, témoignages...). Enfin, le dernier, peut-être le plus marquant et en même temps sans doute le plus difficile à satisfaire, tient à l'absence, pour l'heure, mais la collection n'est pas encore achevée, d'un grand volume 
introductif, qui se situe en amont de 800 et de Charlemagne (que l'on ne saurait considérer comme le "père » de l'histoire franco-allemande...), qui prenne en diagonale l'ensemble des 11 volumes pour justifier ou critiquer l'ambition d'une histoire binationale, pour en montrer les limites mais aussi les points de connexion avec une histoire européenne d'abord, globale ensuite, pour déconstruire les grands récits mis en place entre les deux pays depuis au moins l'époque des Lumières, pour démonter les téléologies auxquelles l'élan de la réconciliation de la deuxième moitié du XXe siècle a pu convier.

7 Ces regrets, pour autant, ne sont pas rédhibitoires et n'enlèvent rien à la valeur ajoutée incontestable et durable fournie par cette entreprise de longue haleine, et peut-être ces lacunes peuvent-elles encore être comblées si l'idée et le courage venaient aux éditeurs de prolonger cette aventure au-delà de son terme éditorial, prévu en 2014, par une sorte de volume encyclopédique raisonné mêlant atlas, lexique, recueil de textes et choix iconographique. Il n'empêche: après les trois volumes du manuel d'histoire francoallemand bilingue destiné aux classes françaises et allemandes de seconde, de première et de terminale publiés entre 2006 et 2011 aux éditions Nathan et Klett, le public scolaire et universitaire, mais aussi le lecteur éclairé soucieux de comprendre sa propre histoire au prisme du regard de l'autre, disposent désormais d'une panoplie complète de manuels et d'ouvrages écrits dans un échange scientifique qui certes n'a pas commencé seulement après 1945, mais qui s'est accéléré et enrichi avec la construction européenne et la réconciliation franco-allemande scellée par la signature du traité de l'Élysée en 1963 dont le cinquantenaire sera célébré en 2013. Fidèles à leur rôle de précurseurs, les historiens des deux côtés du Rhin ont donc fourni par cette majestueuse collection une contribution de taille à un dialogue qui ne saurait avoir valeur de modèle, mais présente du moins un caractère suffisamment exemplaire et une plus-value suffisamment comparative pour constituer la condition d'une entente raisonnée de nos différences et de nos ressemblances.

\section{AUTEUR}

\section{PIERRE MONNET}

Pierre Monnet est directeur de l'IFHA. 